\title{
Changes in age distribution of hemorrhagic fever with renal syndrome: an implication of China's expanded program of immunization
}

\author{
Xiaozhou $\mathrm{He}^{\dagger}$, Shiwen Wang ${ }^{\dagger}$, Xiaoxia Huang and Xiaofang Wang ${ }^{*}$
}

\begin{abstract}
Background: Vaccination against hemorrhagic fever with renal syndrome (HFRS) has been applied successfully for more than 20 years in China, and since 2008, the government has implemented the Expanded Program of Immunization (EPI) in regions with high incidence. In this study, we analyzed the EPI-related changes in age distribution in reported cases of HFRS and proposed new recommendations for prevention and control of the disease.

Methods: Data relating to incidence of HFRS, geographical location and age distribution were collected through the China Information System for Disease Control and Prevention (CISDCP) from 2005 to 2010. Excel and SPSS 18.0 software, $\chi 2$ tests and descriptive methodology were used for analysis of the data.

Results: A total 75434 HFRS cases were reported in 28 provinces in China between 2005 and 2010. The majority of HFRS cases occurred in adults aged 30 to 55 and this group accounted for $68.3 \%$ of the total. With the implementation of the immunization program, HFRS age distribution has clearly changed in recent years. The proportion of HFRS cases among individuals targeted by EPI (16-60 years of age) decreased from 86.9\% in 2005 to 81.9\% in 2010. However, the proportion of cases among the non-vaccinated group aged $<16$ and $>60$ had increased from $13.1 \%$ in 2005 to $18.1 \%$ in 2010. Notably, in the >60 age group the proportion rose from 8.8\% in 2005 to $14.7 \%$ in 2010. These differences were statistically significant.

Conclusion: HFRS vaccination has played an important role in HFRS control and prevention in China. However, since the proportion of HFRS cases over 60 years old has increased significantly since EPI was implemented, it is recommended that the age limit for vaccination be reconsidered. This finding may have practical implications for more effective HFRS vaccination in the future.
\end{abstract}

Keywords: Hemorrhagic fever with renal syndrome, Expanded program of immunization, Age distribution, China

\section{Background}

Hemorrhagic fever with renal syndrome (HFRS), a rodentborne viral disease caused by Hantaviruses, is characterized by fever, hemorrhagic manifestations and renal dysfunction $[1,2]$. The Hantaviruses are usually transmitted to humans via contact with rodent excretions such as saliva, feces and urine [1]. There are two main species of Hantavirus in China: the Hantaan virus (HTNV) and the Seoul virus (SEOV) [2]. China has reported over 1.5 million cases of HFRS since 1950 [3], and HFRS was classified as a Class B

\footnotetext{
* Correspondence: wangxiaofang90@163.com

${ }^{\dagger}$ Equal contributors

National Institute for Viral Disease Control and Prevention, China CDC, Beijing, China
}

notifiable communicable disease in China in 1989. Since then, the number of annual cases throughout the country has been recorded in the national health database that is available for epidemiological study. Up until the end of 2010, all provinces of China, except Hainan province, had reported HFRS cases [4-6]. The annual number of cases in China during the last two decades constituted approximately $90 \%$ of the total cases reported worldwide. But the number of HFRS cases in China has experienced a steady decline in recent years [5,7]. By 2009, the nationwide number of reported cases (8842 cases) had reached its lowest level in nearly two decades.

Aimed at two species of Hantavirus (HTNV and SEOV), a safe and effective bivalent HFRS vaccine was developed

\section{Biomed Central}

(c) 2013 He et al.; licensee BioMed Central Ltd. This is an Open Access article distributed under the terms of the Creative Commons Attribution License (http://creativecommons.org/licenses/by/2.0), which permits unrestricted use, distribution, and reproduction in any medium, provided the original work is properly cited. 
in the 1990s [8] and has been successfully applied [9-12]. In 2008, the HFRS-targeted Expanded Program on Immunization (EPI) was implemented by the Chinese Central Government in order to reduce the HFRS incidence further. Free vaccine was provided to the seven provinces with the highest incidence, namely Heilongjiang, Jilin, Liaoning, Shandong, Hebei, Shaanxi and Zhejiang. One year later, in 2009, HFRS rates were found to have significantly decreased in EPI regions, and the vaccinationbased program was further expanded to 10 other provinces: Hunan, Jiangxi, Inner Mongolia, Hubei, Henan, Jiangsu, Fujian, Anhui, Sichuan and Guangdong. As the number of cases among the 16 to 60 age group accounted for more than 80 percent of the total [13,14], and because the Pharmacopoeia of People's Republic of China (2005) [15] specified that the vaccines could only be used in persons between 16 and 60 years old, the program focused on this age group [16]. However, the bivalent HFRS vaccine is also considered safe for other age groups [10].

In this study, we analyzed the reported HFRS case data from all 17 EPI regions and analyzed the changes in age distribution among the group targeted by EPI (16-60 years of age) and in the non-targeted groups $(<16$ and $>60$ years of age).

\section{Methods}

\section{Data collection and statistical analysis}

Data were obtained from the China Information System for Disease Control and Prevention (CISDCP) and provincial monitoring reports of previous years. This system, established in 2004, is a web-based reporting system of nationally notifiable infectious diseases and public health emergencies throughout the country $[17,18]$. The HFRS cases were diagnosed in the hospital or the local CDC according to the Diagnostic Criteria for Epidemic Hemorrhagic Fever. Two types of cases were analyzed in our study; clinically diagnosed patients and laboratory confirmed cases. Clinical diagnosis criteria included: exposure history (i.e. direct or indirect exposure to rodents and their excreta and saliva within two months prior to onset of illness); and acute onset of disease with at least two clinical symptoms (i.e. hemorrhagic fever, and one or more of the signs of hypotension, oliguresis or renal damage). Laboratory confirmed cases were diagnosed as positive using one of the relevant laboratory tests (HFRS IgM antibody positive, 4 fold rise of IgG antibody between acute and convalescent phage, Hantavirus RNA positive or isolation of Hantavirus from the patient) [19]. Individual case histories of the HFRS patients were collected via the CISDCP according to the Nationwide Surveillance Program for HFRS (try out) [20]. We obtained the following variables for HFRS cases from 2005 to 2010: gender, date of onset, date of birth and residential address.
Analysis was carried out in Microsoft Excel. We calculated the age at onset by using date of onset and date of birth. Then we categorized the data into $<16$ years, $16-$ 60 years and $>60$ years age groups by province. HFRS annual incidence per 100000 population for the nation and each province were collected from the Annual Epidemic Report which is provided by CISDCP as the Summary Analysis of the Epidemic. Demographic data was collected from the Basic Information System, a subsystem of the CISDCP. The number of cases was evaluated by the $x 2$ test in SPSS software to determine the statistical significance of the differences in proportions observed before and after the introduction of the vaccine. Since the coverage of CISDCP was not fully extended to the township level in some western parts of China, only $82.2 \%$ of township hospitals were covered in 2008 [21]; at the time of writing more than $85 \%$ have now been covered but these data are unpublished. Although some cases may not be reported, the evaluations on the quality of the reporting system have shown that the effect of missing reports has been reduced as much as possible [22,23].

\section{Database access permission statement}

The initial disease data which were obtained from the China Information System for Disease Control and Prevention (CISDCP) that is open to the staff of China CDC. All the authors were authorized to obtain and analyze these data, and to publish the results.

\section{Results}

\section{HFRS age distribution in china from 2005 to 2010}

The total number of HFRS cases from 2005 to 2010 in China included in our analyses was 75434 . Cases were reported in 28 provinces, autonomous regions and municipalities; no cases were reported in the Xizang autonomous regions, Qinghai or Hainan provinces during these

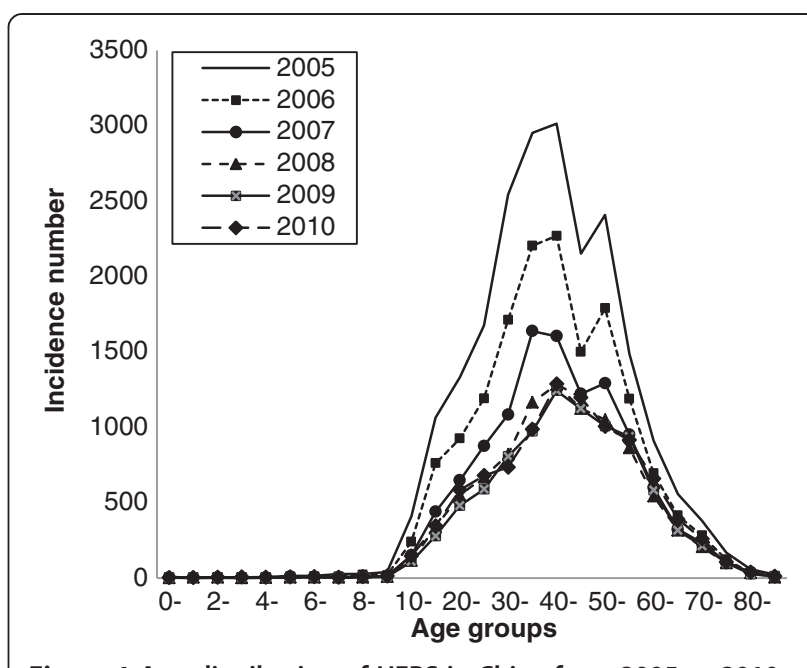

Figure 1 Age distribution of HFRS in China from 2005 to 2010. 
Table 1 HFRS cases among the EPI-targeted age group and non-targeted age group in 2005-2010 for all provinces in China

\begin{tabular}{lllll}
\hline & $\begin{array}{c}\text { Total number } \\
\text { of cases }\end{array}$ & \multicolumn{3}{c}{ Number of cases } \\
\cline { 3 - 5 } Year & \multicolumn{16}{c}{$\mathbf{1 6 - 6 0}$} & $18501(86.9)$ & $911(4.3)$ & $1869(8.8)$ \\
\hline 2005 & 21281 & $13479(87.4)$ & $566(3.7)$ & $1386(9.0)$ \\
2006 & 15429 & $9790(87.0)$ & $302(2.7)$ & $1156(10.3)$ \\
2007 & 11248 & $7890(85.5)$ & $254(2.8)$ & $1083(11.7)$ \\
$2008^{*}$ & 9227 & $7497(84.8)$ & $226(2.6)$ & $1119(12.7)$ \\
$2009^{*}$ & 8842 & $7703(81.9)$ & $322(3.4)$ & $1382(14.7)$ \\
$2010^{*}$ & 9407 &
\end{tabular}

Note. *EPI conducted; $+E P I$-targeted. Data presented as\% of total HFRS cases/yr.

six years. The ratio of male to female cases was approximately 3.15:1 (57 272/18 162); there nas been no change in this proportion in recent years. The majority of HFRS cases occurred in adults aged 30 to 55 (68.3\%; 51 527/75 434). This pattern was similar in all six years evaluated (Figure 1).

The proportion of HFRS cases among individuals targeted by EPI (16-60 years of age) showed a decrease with each year that the immunization program was conducted (Table 1). The proportion of HFRS cases in the non-EPI-targeted (non-immunized) age group increased significantly during the EPI years.
The $\chi^{2}$ test results for HFRS annual number of cases of all provinces for the EPI-targeted and non-targeted age groups indicated that there was no significant difference in number of cases during the years between 2005 and $2007(\chi 2=1.449, P=0.485)$, prior to the implementation of the EPI program. In contrast, a significant difference was found when cases from any EPI year (20082010) were compared with those from any non-EPI year $(2005-2007)(\chi 2=51.095, \mathrm{P}<0.05)$.

The data evaluated from 2005-2010 included the two non-vaccination age groups: children and early- to mid-adolescents ( $<16$ years), and older adults ( $>60$ years). When the $<16$ age group HFRS cases were compared between non-EPI years (2005-2007) and EPI years (20082010), it was found that the numbers did not change significantly $(P=0.142)$. In contrast, the number of HFRS cases in the $>60$ age group was significantly different $(\mathrm{P}<0.05)$ between non-EPI and EPI years. HFRS cases in the older non-vaccinated population ( $>60$ years) rose from $8.8 \%$ in 2005 to $14.7 \%$ in 2010.

When the nationwide HFRS cases were split among the high-incidence, EPI-targeted provinces and the low-incidence, non-EPI provinces, it was found that they displayed different age distribution patterns during the study period. The proportion of HFRS reported cases for the 16-60 age groups actually rose

Table 2 HFRS cases among EPI-targeted age group and non-targeted age group from 2005-2010 in Non-EPI and EPI provinces

\begin{tabular}{|c|c|c|c|c|c|c|}
\hline & \multirow[b]{2}{*}{ Year } & \multirow{2}{*}{$\begin{array}{l}\text { Total number } \\
\text { of cases }\end{array}$} & \multirow{2}{*}{$\begin{array}{l}\text { Incidence per } \\
100000 \text { population }\end{array}$} & \multicolumn{3}{|c|}{ Number of cases (\%) } \\
\hline & & & & $16-60$ years of age & $<16$ years of age & $>60$ years of age \\
\hline \multirow[t]{6}{*}{ Non-EPI provinces } & 2005 & 669 & 0.22 & 614(91.8) & $21(3.1)$ & $34(5.1)$ \\
\hline & 2006 & 432 & 0.14 & 392(90.7) & $16(3.7)$ & 24(5.6) \\
\hline & 2007 & 230 & 0.08 & $201(87.4)$ & $10(4.3)$ & $19(8.3)$ \\
\hline & 2008 & 219 & 0.07 & 193(88.1) & $8(3.7)$ & $18(8.2)$ \\
\hline & 2009 & 199 & 0.06 & 165(82.9) & $9(4.5)$ & 25(12.6) \\
\hline & 2010 & 194 & 0.06 & 169(87.1) & $5(2.6)$ & $20(10.3)$ \\
\hline \multirow[t]{12}{*}{ Provinces targeted for EPI since 2008} & 2005 & 17402 & 4.93 & $15177(87.2)$ & $742(4.3)$ & 1483(8.5) \\
\hline & 2006 & 12137 & 3.42 & $10680(88.0)$ & 436(3.6) & $1021(8.4)$ \\
\hline & 2007 & 8374 & 2.34 & 7394(88.3) & $208(2.5)$ & $772(9.2)$ \\
\hline & $2008^{*}$ & 6959 & 1.93 & 5975(85.9) & $184(2.6)$ & $800(11.5)$ \\
\hline & $2009^{*}$ & 6378 & 1.76 & $5464(85.7)$ & 166(2.6) & 748(11.7) \\
\hline & $2010^{*}$ & 6995 & 1.92 & 5709(81.6) & $261(3.7)$ & $1025(14.7)$ \\
\hline & 2005 & 3210 & 0.51 & $2711(84.5)$ & $148(4.6)$ & $351(10.9)$ \\
\hline & 2006 & 2860 & 0.46 & 2408(84.2) & 112(3.9) & $340(11.9)$ \\
\hline & 2007 & 2644 & 0.42 & 2195(83.0) & $84(3.2)$ & $365(13.8)$ \\
\hline & 2008 & 2049 & 0.32 & 1723(84.1) & $62(3.0)$ & 264(12.9) \\
\hline & $2009^{* *}$ & 2265 & 0.36 & $1867(82.5)$ & $52(2.3)$ & $346(15.3)$ \\
\hline & $2010^{* *}$ & 2218 & 0.35 & 1825(82.3) & $56(2.5)$ & $337(15.2)$ \\
\hline
\end{tabular}


(from $82.9 \%$ in 2009 to $87.1 \%$ in 2010 ) in the non-EPI regions (Table 2).

\section{Analysis of high-incidence, EPI-targeted provinces}

Despite the implementation of EPI, HFRS cases have remained mainly concentrated in Northeast and East China, where the seven high-incidence provinces are located. In 2010, these seven provinces accounted for 74.3\% (6978/ 9407) of the total cases reported in China, and this situation had changed very little over the other five years: $81.7 \%$ in 2005 (17 383/21 281), 78.6\% in 2006 (12 129/15 429), $74.5 \%$ in 2007, (8380/11 248), $75.4 \%$ in 2008 (6958/ $9227)$ and $72.2 \%$ in $2009(6380 / 8842)$.

Over the last three years the number of HFRS cases in Shaanxi province has steadily increased. In 2010, the total annual number of reported cases (2356) had increased by $65 \%$ over the 2009 case number (1428), and had the highest number of HFRS cases in any year since 2005 [5]. This might be partly explained by well-known periodic changes in zoonoses and some social or environmental factors. Like other high-incidence provinces, the proportion of cases in the 16-60 age groups has declined since 2008 when EPI was first implemented in Shaanxi. However, the proportion of HFRS cases among the $>60$ age group has been substantially higher than in other high-incidence provinces. From 2009 to 2010 the
HFRS cases in $>60$ year old Shaanxi residents increased by $67.2 \%$ (413 to 247) (Figure 2).

Heilongjiang, Jilin, Liaoning, Shandong, Hebei and Zhejiang provinces showed a steady decline in total HFRS cases since 2005, but the slope of this downward trend has decreased in recent years. Moreover, the incidence per 100000 population in Liaoning, Shandong and Zhejiang provinces rebounded slightly in 2010. In each of these six provinces, the proportion of cases in 2010 among the respective 16-60 age groups decreased (ranging from $0.7 \%$ in Heilongjiang to a high of $8.1 \%$ in Shandong) and the proportion of cases in the $>60$ age groups increased (ranging from 26.7\% in Zhejiang to $45.9 \%$ in Liaoning). The proportion of HFRS cases among the respective $<16$ age groups did not experience any obvious changes in the years examined (Figure 2).

\section{Discussion}

As a zoonosis, HFRS shows periodic variations, which may be one of the reasons why the number of cases decreased in the years 2005-2007. HFRS has also been shown to be associated with geographical locale, employment type, living (hygiene) conditions and the epidemiology of the rodent-vector $[3,24,25]$. The national implementation of a comprehensive prevention and control strategy, involving rodent control, environmental management and
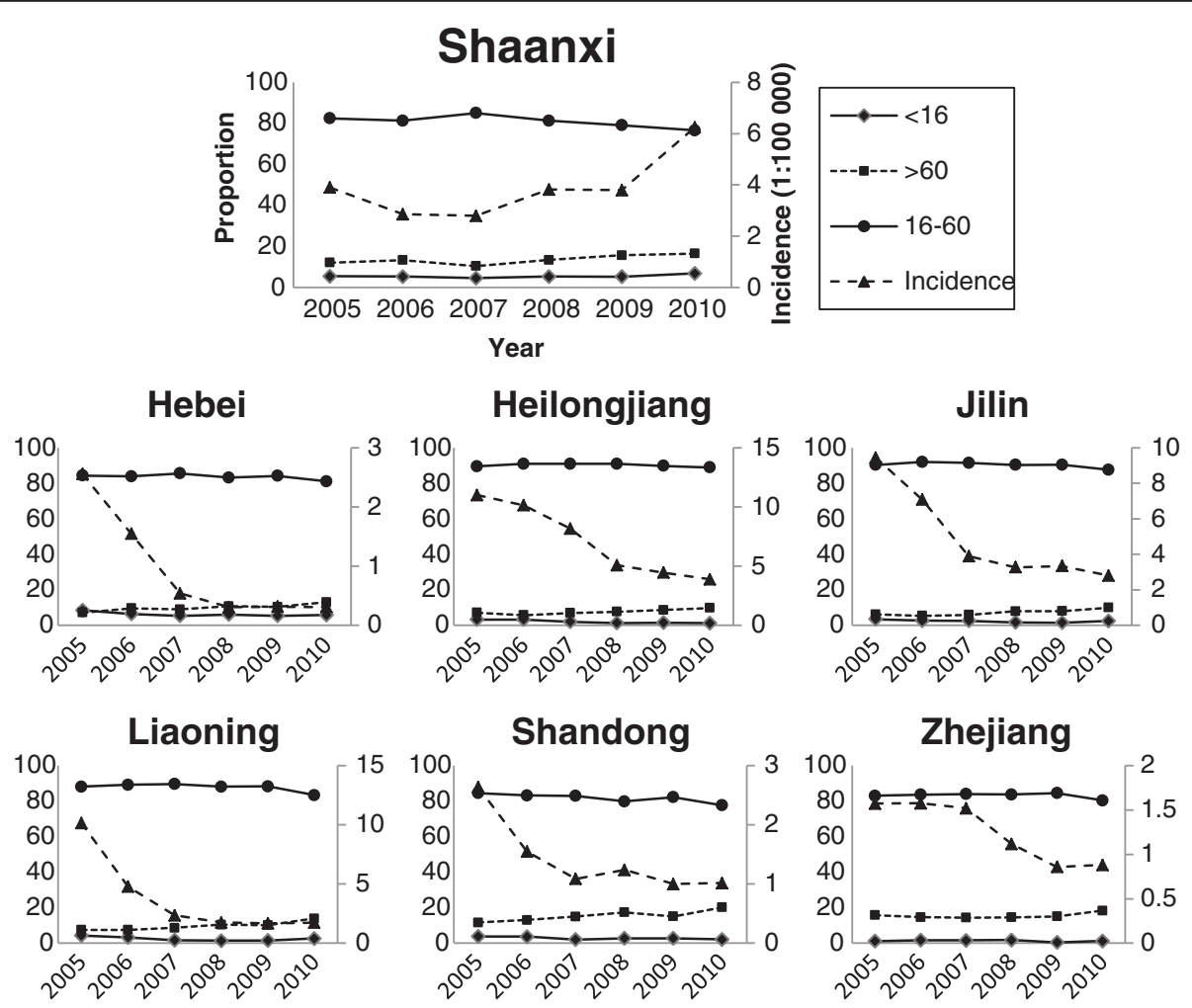

Figure 2 Proportion of HFRS among different age groups and the incidence per 100000 population per province in seven EPI-target provinces from 2005 to 2010. 
vaccination, has corresponded to a remarkable decline in incidence of HFRS in China.

In order to reduce the incidence further, the EPI was implemented in 2008. When the proportions of cases among the different HFRS age groups were analyzed, it was found that the age distribution had changed in recent years in the EPI regions. The group that was targeted by EPI (16-60 years of age) experienced a significant decrease in the proportion of HFRS cases for each year that EPI was implemented, suggesting that vaccination might have played a considerable role in China's HFRS prevention and control. Further analysis revealed that the proportion of cases in children less than 16 years old was relatively unaffected during this period. Moreover, this stable profile remained when only the $<16$ years old, non-EPI-targeted HFRS cases were analyzed $(<10$ years old vs. 10-16 years old, for all years examined; data not shown).

We were intrigued to discover, however, that the proportion of HFRS cases in the population over 60 years old in EPI-targeted provinces had increased annually from 2008 to 2010. In fact, Shandong province had $20.2 \%$ of its HFRS cases in the $>60$ age group in 2010. In stark contrast, the proportion of cases in the over 60 age group residing in non-EPI-targeted provinces had declined in 2010.

The observation that the proportion of HFRS cases over 60 years of age has risen in EPI-targeted regions may be explained by the following reasons. In recent years, more young people from rural areas have travelled to the cities for work, increasing the necessity for the elderly who have stayed behind to spend longer hours working in the fields. The elderly then represent the population most in contact with rodents and potential transmission of Hantavirus. Furthermore, the gradual extension of average life expectancy may have increased the proportion of individuals in the $>60$ age group who are still alive and working and potentially coming into contact with Hantaviruses.. Another potential explanation may be that China Pharmacopoeia defined the HFRS bivalent vaccine-target group as 16-60 years old, and over the dozen years since HFRS vaccination the current $>60$ years age group might not have had sufficient opportunity for immunization.

\section{Conclusions}

The recent trend showing an increase in the proportion of HFRS cases in individuals over 60 years of age has occurred in regions undergoing rodent-based prevention strategies and environmental remediation and in which comprehensive public health education is widely available. Unfortunately, these non-vaccine based eradication strategies have not been sufficient to protect this older population from HFRS. Therefore, we recommend that the age limit for EPI vaccination be extended beyond 60 years old, as we believe it would be beneficial to HFRS control and prevention in China.

Competing interests

The authors declare that they have no competing interests.

\section{Authors' contributions}

$\mathrm{XH}$ was involved in data collection, data analysis and drafting of the manuscript. SW conceived and designed the study, also participated in interpreting the data. $\mathrm{XH}$ assisted with reviewing and revision the manuscript. XW conducted the analysis and drafted the manuscript. All authors read and approved the final manuscript.

\section{Acknowledgements}

This research was supported by China Mega-Project for Infectious Diseases grants (No. 2012ZX10004215) from Ministry of Science and Technology and the Ministry of Health.

Received: 23 October 2012 Accepted: 19 April 2013

Published: 26 April 2013

\section{References}

1. Lee PW, Amyx HL, Gibbs CJ Jr, Gajdusek DC, Lee HW: Propagation of Korean hemorrhagic fever virus in laboratory rats. Infect Immun 1981, 31(1):334-338

2. Wang SW, Hang CS, Wang H, Xie YX, Ma BJ: Genotype and clade distribution of hantaviruses in china. Chin J Virol 2002, 18(3):211-216.

3. Zhang YZ, Zou Y, Fu ZF, Plyusnin A: Hantavirus infections in humans and animals, China. Emerg Infect Dis 2010, 16(8):1195-1203.

4. Liu X, Jiang B, Bi P, Yang W, Liu Q: Prevalence of haemorrhagic fever with renal syndrome in mainland China: analysis of National Surveillance Data, 2004-2009. Epidemiol Infect 2012, 140(5):851-857.

5. Wang Q, Zhou H, Han YH, Wang XF, Wang SW, Yin WW, Li Q, Xu Z: Epidemiology and surveillance programs on hemorrhagic fever with renal syndrome in Mainland China, 2005-2008. Zhonghua liu xing bing xue za zhi= Zhonghua liuxingbingxue zazhi 2010, 31(6):675-680.

6. Xiao-fang W, Mao-wu W, Hui S: Epidemiological analysis of hemorrhagic fever with renal syndrome in China from 2004 to 2005. Dis Surveliance 2007, 22(5):307-309.

7. Xiaoxia H, Hongmei Y, Lei Y, Xiaofang W, Shiwen W: Epidemiologic characteristics of haemorrhagic fever with renal syndrome in mainland china from 2006 to 2010. West Pac Surveillance Response 2012, 3(1):12-18.

8. Hang C, Xie Y, Wang S, Huo Z, Zhang Q: Advances on development of purified bivalent vaccine against Epidemic Hemorrhagic Fever (HFRS) prepared on Vero cells. Bull Med Res 2001, 5:2-4.

9. Gong ZY, Weng JQ, Lei JB, Fang CF, Chen EF, Wang Z, Chen ZB, Wang W, He F, Liu BY, et al: Study on a 10-year protective effects of vaccination against hemorrhagic fever with renal syndrome. Zhonghua liu xing bing xue za zhi = Zhonghua liuxingbingxue zazhi 2007, 28(12):1190-1193.

10. Dahan $L$ : An observation of HFRS bivalent vaccine safety and immunogenicity. Jiangsu J Preventive Med 2008, 4:18-19.

11. Yang $C$, Shiqing $L$, Si $H$, Changping $C$, Renyi $Y$, Anping $Z$ : A Preliminary observation epidemiological results for HFRS bivalent vaccine. Strait J Preventive Med 2006, 1:28-30.

12. Changying C: Effectiveness evaluation and immunization strategies for HFRS bivalent vaccine. Chin J Epidemio/ 2003, 2:86-87.

13. Luo CW, Chen HX: Epidemiological characteristics and the strategy of vaccination on hemorrhagic fever with renal syndrome in the last 10 years, in China. Zhonghua liu xing bing xue za zhi= Zhonghua liuxingbingxue zazhi 2008, 29(10):1017-1019.

14. Chang-an M, Zhanzhen W, Zhi-hu Q, Jian-hui Q, Xin T: Analysis of HFRS Epidemiology characteristic after Immunization in Hu- country. Chin $J$ Vector Biol Control 2008, 6:567-569.

15. National Pharmacopoeia Committee: Pharmacopoeia of the People's Republic of China, the third division of 2005 edition. Beijing: China Chemical Industry Press; 2005:80-88

16. Ministry of Health of the People's Republic of China: National Implementing Scheme of Expanded Program on Immunization. 2008. http://www.moh.gov. cn/wsb/s7374/200806/36398.shtml. 
17. Xiong W, LV J, Li L: A survey of core and support activities of communicable disease surveillance systems at operating-level CDCs in China. BMC Publ Health 2010, 10:704.

18. Wang L, Wang Y, Jin S, Wu Z, Chin DP, Koplan JP, Wilson ME: Emergence and control of infectious diseases in China. Lancet 2008, 372(9649):1598-1605.

19. Ministry of Health of the People's Republic of China: Diagnostic criteria for epidemic hemorrhagic fever (HFRS). 2008. http://www.moh.gov.cn/zwgkzt/ s9491/200902/39043.shtml.

20. Ministry of Health of the People's Republic of China: Nationwide Surveillance Program for HFRS (try out). 2005. http://www.ivdc.chinacdc.cn/jbkz/jsfa/ 201110/P020111017505587180289.pdf.

21. Liu SW, Wang LP, Wang XF, Zhang CX, Guo Q, Zhou MG, MA JQ: Evaluation on management and quality of communicable diseases network direct reporting in China. Dis Surveillance 2009, 2011(5):392-397.

22. Wang LJ, Ma JQ, Zhou MG, Wang YY, Ge H, Yang GH: Study on the evaluation of underreporting and the quality of death cases reporting system, from medical institutions at county level and above, in, 2005. Zhonghua liu xing bing xue za zhi = Zhonghua liuxingbingxue zazhi 2007, 28(6):571-575.

23. Wang LP, Guo Y, Guo Q, ZHANG CX: Evaluation on quality of internet-based reporting of notifiable communicable diseases in China, 2005-2008. Dis Surveillance 2010, 11:912-914.

24. Guo HQ, Ding HL, Bo Q, Sun G: Epidemic information of hemorrhagic fever with renal syndrome in China from 2004 to 2009 in ARIMA model. Chin J Zoonoses 2010, 26(12):1137-1140.

25. Yan L, Fang LQ, Huang HG, Zhang LQ, Feng D, Zhao WJ, Zhang WY, Li XW, Cao WC: Landscape elements and Hantaan virus-related hemorrhagic fever with renal syndrome, People's Republic of China. Emerg Infect Dis 2007, 13(9):1301-1306.

doi:10.1186/1471-2458-13-394

Cite this article as: He et al:: Changes in age distribution of hemorrhagic fever with renal syndrome: an implication of China's expanded program of immunization. BMC Public Health 2013 13:394.

\section{Submit your next manuscript to BioMed Central and take full advantage of:}

- Convenient online submission

- Thorough peer review

- No space constraints or color figure charges

- Immediate publication on acceptance

- Inclusion in PubMed, CAS, Scopus and Google Scholar

- Research which is freely available for redistribution 